\title{
Flora del Parque Arqueológico de Copán, Honduras
}

Lilian Ferrufino-Acosta, German Sandoval y Olvin Oyuela ${ }^{1}$

\section{RESUMEN}

El Parque Arqueológico de Copán se estudió la flora que habita en esta área protegida. Se registraron 94 especies vegetales, siendo el $76 \%$ eudicotiledóneas, 16\% monocotiledóneas y el $10 \%$ angiospermas basales. La familia Fabaceae (10) es la mejor representada. En los nueve transeptos lineales de $0.1 \mathrm{~km}$ se identificaron un total de 57 especies de plantas arbóreas y arbustivas y se contaron 605 individuos, siendo Brosimum alicastrum Sw. el más abundante. Las especies Cedrela odorata L. y Swietenia macrophylla King están categorizadas en su estado de conservación como vulnerable según la lista roja de UICN y el apéndice III de CITES. En su mayoría, las especies registradas en el sitio presentan un uso potencial ya sea medicinal, industrial y de aprovechamiento forestal.

Palabras clave: sitio arqueológico, especies vegetales, Fabaceae, Brosimum alicastrum.

${ }_{1}^{1}$ Profesores de la Escuela de Biología y Herbario Cyril Hardy Nelson Sutherland (TEFH), Facultad de Ciencias, UNAH: lilian.ferrufino@unah.edu.hn, german.sandoval@unah.edu.hn, olvin.oyuela@unah.edu.hn. 


\section{ABSTRACT}

The Archaeological Park of Copan studied the flora that inhabit in this protected area. There were 94 plant species, being $76 \%$ eudicotiledóneas, 16\% monocotyledons and $10 \%$ basal angiosperms. The Fabaceae family (10) has the highest number of species. In the nine linear transects of $0.1 \mathrm{~km}$, a total of 57 species of arboreal and shrub plants were identified and 605 individuals were counted, being the most abundant Brosimum alicastrum. Cedrela odorata $L$. and Swietenia macrophylla species categorized as vulnerable according to the IUCN red list and listed in Appendix III of CITES. Most of the species registered on the site present a potential use, as medicinal, industrial and forestry.

Key words: Archaeological sites, plant species, Fabaceae, Brosimum alicastrum. 


\section{INTRODUCCIÓN}

El Parque Arqueológico de Copan fue declarado por la UNESCO Patrimonio Mundial el 5 de septiembre de 1980, siendo uno de los sitios más turísticos en Honduras. Este territorio fue parte, junto con el sur de México y Guatemala, del "Área Maya del Pacífico", considerándose un centro de domesticación de plantas y aves para huertos familiares. Actualmente, el territorio es cohabitado por los herederos de los mayas y mestizos; de forma tal que la hibridación de estas culturas conlleva el cultivo y la crianza de plantas y animales nativos e introducidos (Rivas 2010). Según Vargas Pérez (2004) el área maya mesoamericana se divide tres regiones: 1) la norte, que abarca Yucatán, el norte de Campeche y Quintana Roo en México; 2) el área central, incluye el Palenque en México, Petén en Guatemala hasta Copán en Honduras; y la 3) el área sur, que incluye los altiplanos y costas del Océano Pacífico de Chiapas y Guatemala y las áreas costeras de El Salvador y Honduras.

La vegetación del El Parque Arqueológico de Copán está formada por bosque secundario con una alta densidad de árboles de Brosimum alicastrum Sw. (san ramón). Sin embargo, la presencia de otras especies como Hymenaea courbaril L. (guapinol), Bursera simaruba (L.) Sarg. (indio desnudo), Enterolobium cyclocarpum (Jacq.) Griseb. (guanacaste), Cedrela odorata L. (cedro). Además está conformada por un bosque deciduo estacional mixto constituido por árboles que dejan caer sus hojas en la estación seca (House 2007; Asociación Copán 2013). Sin embargo, en la periferia del parque las actividades antrópicas como la ganadería y la agricultura han contribuido a la deforestación del área.

Los bosques estaciónales de Copán en comparación con otros sitios del Mundo Maya son relativamente pequeños y muy fragmentado, y por esta razón su valor ecológico fue subestimado. En estudios recientes a nivel nacional se ha demostrado que estos bosques se encuentran entre los más raros y amenazados de todo Honduras. Siendo el fragmento dentro del área protegida de las Ruinas de Copán el único bosque de esta categoría protegida en Honduras (House 2007).

El propósito de este proyecto fue realizar un inventario florístico del Parque Arqueológico de Copán para evaluar la diversidad vegetal en los fragmentos de bosque y de esta manera contribuir al Programa de Manejo de Recursos Naturales con el fin de cumplir las actividades y las líneas estratégicas propuestas en el plan de manejo 2014-2020. 


\section{Materiales y métodos}

\section{Área de estudio}

El Parque Arqueológico de Copán se encuentra ubicado en el Valle de Copán en el municipio de Copán Ruinas en el departamento de Copán, Honduras. Cuenta con un área de 60.95 ha y se ubica a $89^{\circ} 08^{\prime} 31^{\prime \prime}$ W, 14 $50^{\circ} 22^{\prime \prime} \mathrm{N}$ en una altitud 500-600 msnm (figura 1). En la zona se registra una temperatura promedio anual superior a los $18^{\circ}$ C. y con una precipitación anual promedio superior a los 1,000 mm. (Asociación Copán, 2013).

Figura 1. Localización del Parque Arqueológico de Copán en imagen satelital y de los nueves transeptos basados en las coordenadas.

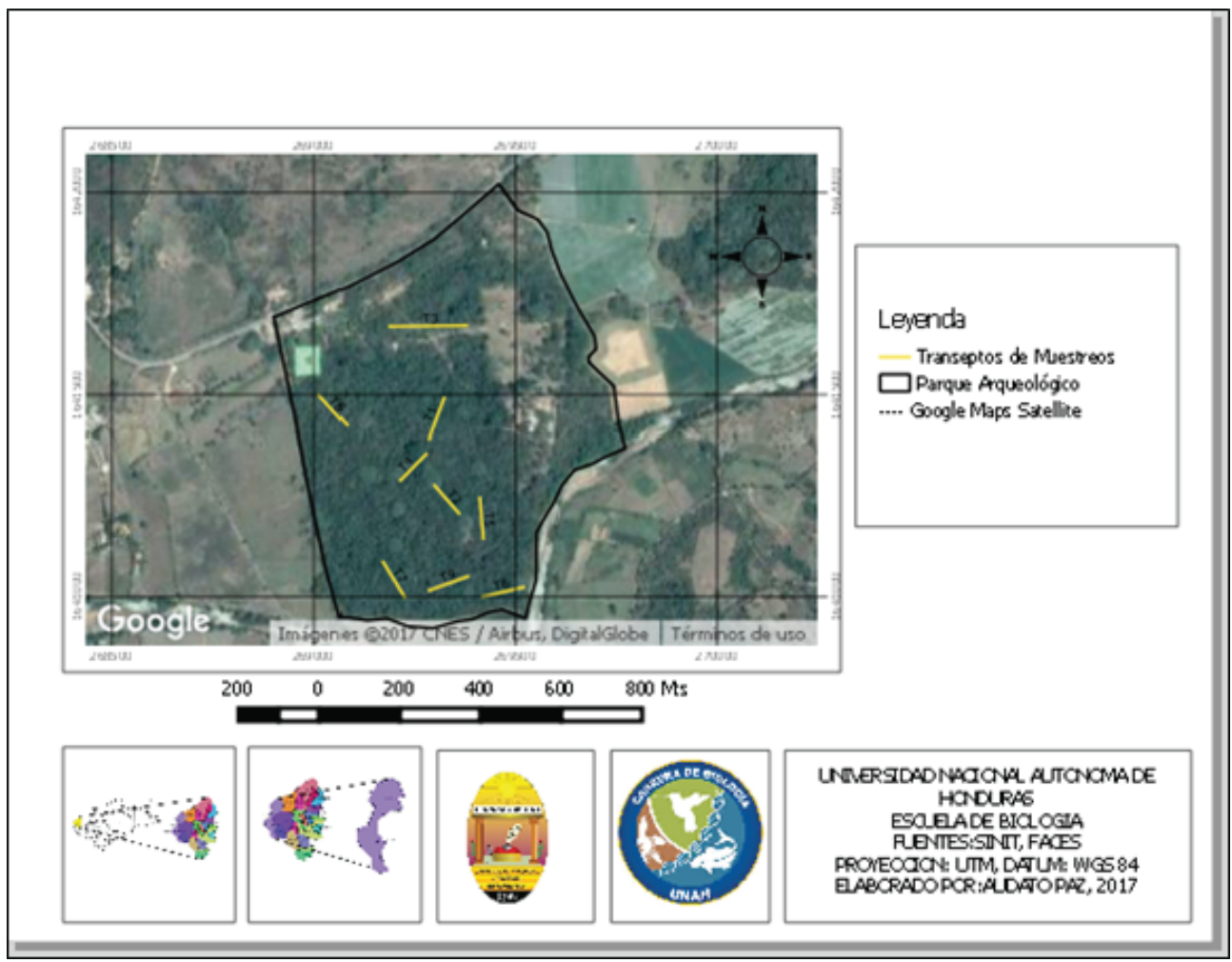




\section{Muestreo}

El estudio fue realizado entre el 25-26 de agosto de 2016 y del 9-10 de junio de 2017. Para determinar la riqueza de especies de plantas leñosas se realizaron nueve transeptos lineales de $0.1 \mathrm{~km}$, según el método Gentry (1982) (figura 1). Cada uno abarcó $100 \mathrm{~m}$ de longitud x $10 \mathrm{~m}$ de ancho, distribuidos en el área boscosa dentro del Parque Arqueológico de Copán (Figura 2). Una vez establecidos y georreferenciados los transeptos, se identificó y se contó el número de individuos por especie. La representación de los datos de densidad de las 48 especies registradas, el área calculada es aproximada de 2,6 ha.

$100 \mathrm{~m} \times 10 \mathrm{~m} 1000 \mathrm{~m}^{2}=0,1$ ha $\times 9$ transeptos $=0.9$ ha

El material vegetal fue prensado, secado e identificado en el Herbario Cyril Hardy Nelson Sutherland (TEFH). Los nombres comunes fueron consultados en el catálogo de plantas vasculares de Honduras (Sutherland Hardy 2008) y otros consultados a los guías de campo.

\section{Resultados y discusión}

De las 94 especies registradas, 67 son eudicotiledóneas (71\%), 15 monocotiledóneas (16\%), 10 angiospermas basales (13\%) y una especie de helecho (cuadro 1 y figuras 2), siendo la mayoría de las especies nativas. Entre las especies exóticas se registra la especie de orquídea terrestre Oeceoclades maculata (Lindl.) Lindl., considerada una especie invasora y actualmente se ha observado que invade áreas de amortiguamiento en reservas naturales del país.

Estudios realizados en sitios arqueológicos de la región Maya registran especies y familias de angiospermas similares en particular eudicotiledóneas a las encontradas en el Parque Arqueológico Copán. Gómez-Domínguez et al. (2015) con un listado florístico del Parque Nacional Palenque en Chiapas, México ... no obstante la comparación con otros estudios realizados con base en el número de especies es difícil ya que Copán es el sitio más pequeña del área Maya.

En los nueve transeptos se identificaron un total de 605 individuos, de estas 57 especies arbóreas y arbustivas. El transepto número ocho presentó la mayor abundancia con 120 individuos, por el contrario el transepto nueve con 37 individuos, siendo el menos abundante (Figura 3). 
Figura 2. Algunas especies registradas en el Parque Arqueológico de Copán.
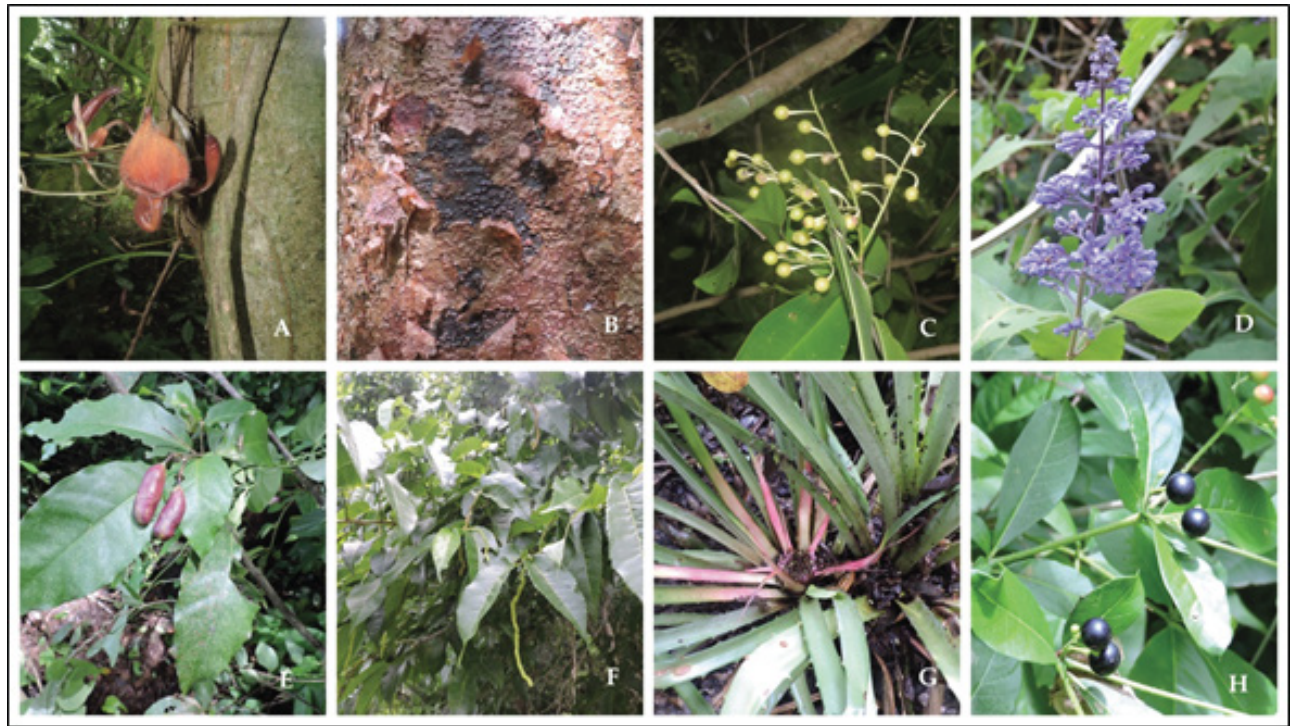

A. Aristolochia máxima Jacq.; B. Bursera simaruba (L.) Sarg.; C. Ardisia compressa Kunth; D. Cornutia pyramidata L.; E. Capparis frondosa Jacq.; F. Maclura tinctoria (L.) D. Don ex Steud.; G. Bromelia karatas L.; H. Rauvolfia tetraphylla

Fuente: fotos tomadas por el personal del Herbario TEFH

Dentro de los nueve transeptos la especie más abundante fue Brosimum alicastrum (san ramón) con un total de 146 individuos y una densidad de 162 individuos por hectárea Cuadro 2). Esta especie forma parte de las forrajeras en las comunidades Mayas en Yucatán, México (Flores y Bautista 2012). En el sitio arqueológico de Oxpemul en Campeche, México B. alicastrum presentó mayor abundancia, considerando que favorece su crecimiento en ambientes que generalmente presentan alta pedregosidad con suelos delgados (Zamora-Crescencio y Gutiérrez-Báez, 2012).

El Valle de Copán presenta llanuras aluviales o vegas y terrazas con pendientes pronunciadas (Asociación Copán 2013). Vega-López et al. (2003) comentan que Brosimum alicastrum en la costa del Océano Pacífico habita en suelos aluviales. 
Figura 3. Número de individuos por transeptos en los sitios muestreados en el Parque Arqueológico de Copán.

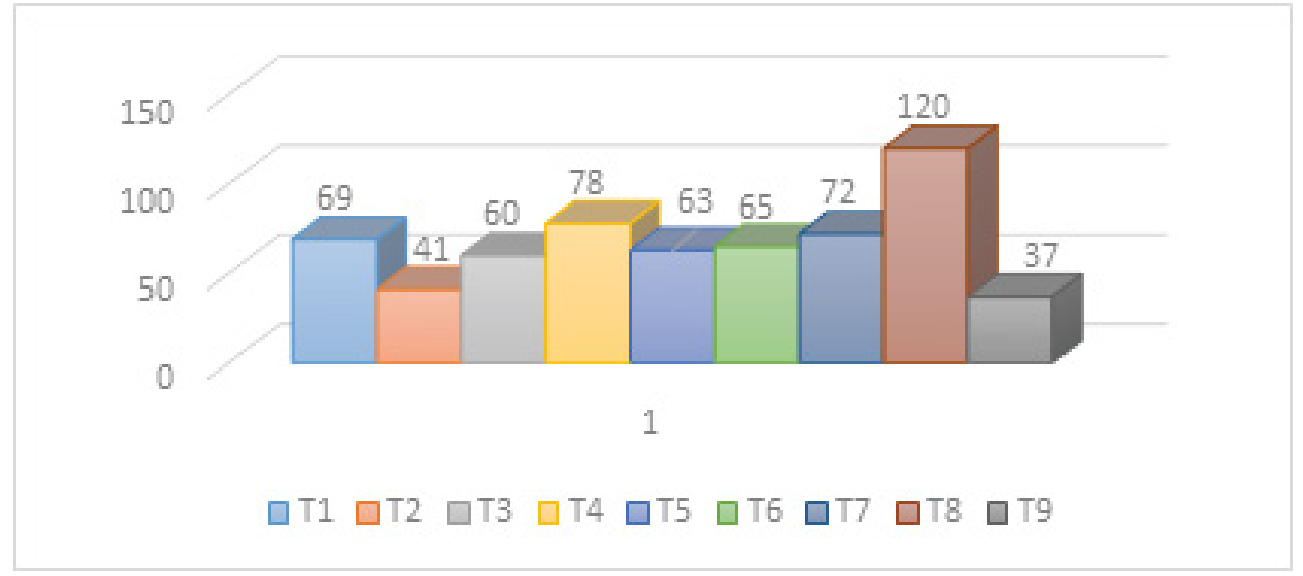

Cuadro 2. Abundancia, porcentaje de cobertura por especies y densidad de las especies registradas en el Parque Arqueológico de Copán.

\begin{tabular}{|l|l|l|l|}
\hline Taxa & Abundancia & $\%$ de cobertura & Densidad \\
\hline Brosimum alicastrum & 146 & 57,0 & 162,2 \\
\hline Ardisia paschalis & 60 & 23,4 & 66,7 \\
\hline Calycophyllum mexicanum & 51 & 19,9 & 56,7 \\
\hline Bursera simaruba & 46 & 18,0 & 51,1 \\
\hline Guazuma ulmifolia & 31 & 12,1 & 34,4 \\
\hline Cedrela odorata & 30 & 11,7 & 33,3 \\
\hline Piper aduncum & 25 & 9,8 & 27,8 \\
\hline Astronium graveolens & 22 & 8,6 & 24,4 \\
\hline Bromelia pinguin & 19 & 7,4 & 21,1 \\
\hline Spondias mombin & 12 & 4,7 & 13,3 \\
\hline Trichillia martiana & 12 & 4,7 & 13,3 \\
\hline Bellucia axinanthera & 11 & 4,3 & 12,2 \\
\hline Pithecoelobium dulce & 11 & 4,3 & 12,2 \\
\hline Eugenia sp. & 10 & 3,9 & 11,1 \\
\hline Pisonia aculeata & 9 & 3,5 & 10,0 \\
\hline Rubus sp. & 9 & 3,5 & 10,0 \\
\hline Cordia alliodora & 7 & 2,7 & 7,8 \\
\hline Thouinia serrata & 7 & 2,7 & 7,8 \\
\hline Urera baccifera & 7 & 2,7 & 7,8 \\
\hline
\end{tabular}




\begin{tabular}{|l|l|l|l|}
\hline Taxa & Abundancia & \% de cobertura & Densidad \\
\hline Annona muricata & 6 & 2,3 & 6,7 \\
\hline Castilla elastica & 6 & 2,3 & 6,7 \\
\hline Enterolobium cyclocarpum & 6 & 2,3 & 6,7 \\
\hline Epiphyllum hookerii & 6 & 2,3 & 6,7 \\
\hline Acacia collinsii & 5 & 2,0 & 5,6 \\
\hline Acrocomia mexicana & 5 & 2,0 & 5,6 \\
\hline Glirididia sepium & 4 & 1,6 & 4,4 \\
\hline Zanthoxylum sp. & 4 & 1,6 & 4,4 \\
\hline Ceiba pentandra & 3 & 1,2 & 3,3 \\
\hline Bonellia macrocarpa & 3 & 1,2 & 3,3 \\
\hline Ceiba petandra & 3 & 1,2 & 3,3 \\
\hline Fabaceae & 3 & 1,2 & 3,3 \\
\hline Myrsinaceae & 3 & 1,2 & 3,3 \\
\hline Morfoespecie1 & 3 & 1,2 & 3,3 \\
\hline Crataeva tapia & 2 & 0,8 & 2,2 \\
\hline Genipa americana & 2 & 0,8 & 2,2 \\
\hline Morfoespecie2 & 2 & 0,8 & 2,2 \\
\hline Morfoespecie3 & 2 & 0,8 & 2,2 \\
\hline Morfoespecie4 & 2 & 0,8 & 2,2 \\
\hline Alvaradoa amorphoides & 1 & 0,4 & 1,1 \\
\hline Andira inermis & 1 & 0,4 & 1,1 \\
\hline Arrabidea sp. & 1 & 0,4 & 1,1 \\
\hline Carludovica palmata & 1 & 0,4 & 1,1 \\
\hline Cecropia peltata & 1 & 0,4 & 1,1 \\
\hline Luehea speciosa & 1 & 0,4 & 1,1 \\
\hline Oncidium cebolleta & 1 & 0,4 & 1,1 \\
\hline Quercus sp. & 1 & 0,4 & 1,1 \\
\hline Swietenia humilis & 1 & 0,4 & 1,1 \\
\hline Trichilia havanensis & 1 & 0,4 & 1,1 \\
\hline & & & \\
\hline
\end{tabular}

\section{Riqueza de especies por familia}

La familia con más riqueza de especies encontrada fue Fabaceae con diez especies, seguida por Meliaceae, Moraceae y Piperaceae con cinco especies cada familia, así como Apocynaceae y Rubiaceae con cuatro especies. El resto de las especies se 
ubican en diferentes familias de Angiopermas y Monilofitas (cuadro 1). En otros sitios arqueológico también ha sido reportada la familia Fabaceae con mayor número de especies: el sitio arqueológico Casa Vieja, en el distrito Callango en Perú (Roque et al., 2003); así como el cono sur del estado de Yucatán (Zamora-Crescencio et al., 2009); y Oxpemul en Campeche, México (Zamora-Crescencio et al., 2012); y en los huertos familiares de Quintana Roo, México (Kantún-Balam et al., 2013) y en las comunidades aledañas a la Reserva de la Biosfera de Calakmul, Campeche, México (Chi Quej, 2009).

\section{Estado de conservación de las especies}

Según la lista Roja de la Unión Internacional para la Conservación de La Naturaleza (UICN) categoriza Lonchocarpus rugosus Benth. (Fabaceae) en preocupación menor. L. rugosus tiene una madera de alta calidad y habita en una variedad de tipos de hábitat, incluyendo bosques de robles bajos, bosques tropicales con Enterolobium, bosque subdeciduo con Brosimum, así como laderas y barrancos. Este taxón está adaptado para hacer frente a las condiciones de sequía (Vozzo 2002).

Otras especies de la familia Meliaceae, Cedrela odorata y Swietenia macrophylla en la categoría vulnerable. La Convención sobre el Comercio Internacional de Especies Amenazadas de Fauna y Flora Silvestres (CITES) ubica las especies de la familia de monocotiledóneas: Orchidaceae y Bromeliaceae y S. macrophylla en el apéndice II; no obstante $C$. odorata la coloca en el apéndice III.

\section{Usos potenciales}

Entre otras las especies de importancia económica en particular en aprovechamiento forestal que se encuentran en el parque, se enlistan: Cedrela odorata, Cordia alliodora, Maclura tinctoria, Swietenia macrophylla, Luehea speciosa, Quercus sp. Enterolobium cyclocarpum, Genipa americana, Ceiba pentandra, Astronium graveolens, Guazuma ulmifolia, Spondias purpurea, Calycophyllum candidissimum, Trichilia martiana, Sapranthus violaceus, Alvaradoa amorphoides, Lonchocarpus rugosus, Simarouba glauca, Pithecellobium dulce.

Otras especies registradas en el sitio con importancia industrial se enlistan: Carludovica palmata, Jatropha curcas, Maclura tinctoria, Trichillia martiana, Castilla elástica, Indigofera suffruticosa, Andira inermis. Algunas comestibles como Spondias purpurea, Annona muricata, Sideroxylon capiri, Genipa americana, Guazuma. ulmifolia, Simarouba glauca, Pithecellobium dulce, Brosimum alicastrum, Acrocomia mexicana. 
También se usan especies para el forraje: Gliricidia sepium, G. ulmifolia, S. purpurea, Ceiba pentandra, Bursera simaruba, P. dulce, B. alicastrum, así como cercas vivas, G. sepium. Flores y Bautista (2012) comentan que las comunidades mayas de la Península de Yucatán en México usan 196 especies vegetales para alimentar a los animales, de las cuales 139 herbáceas, 17 arbustivas, 35 arbóreas y dos palmeras.

La mayoría de las especies del parque presentan propiedades medicinales entre las que se menciona Smilax spinosa, Genipa americana, Guazuma ulmifolia, Jatropha curcas, A. graveolens, Annona muricata, Calycophyllum candidissimum, Cedrela odorata, Ceiba pentandra, Trichillia martiana, Alvaradoa amorphoides, Bursera simaruba, Simarouba glauca, Pithecellobium dulce, Andira inermis, Bonellia macrocarpa, Casearia corymbosa, Hamelia patens, Cornutia pyramidata, Dioscorea sp. Otras especies sus flores producen miel siendo: C. candidissimum, Pithecellobium dulce, C. alliodora. Zamora Crescencio et al. (2009) mencionan que las familias rurales mayas en el Cono Sur del estado de Yucatán reportan de la flora local el uso de plantas medicinales.

Algunas especies nativas han sido cultivadas en el sitio por los mayas como es el caso de Brosimun alicastrum, llamado comúnmente "másica" o "san ramón" como un sustituto del maíz y de papa. Además, los frutos fueron usados por los precolombinos ya que la fruta posee un olor agradable y dulce y a su vez la harina de la semilla fue usada por los mayas. Entro otros usos que se reportan: pastos, usos medicinales y maderables (Cordero y Boshier 2003).

Vargas Pérez (2004) menciona que los mayas han usado los recursos naturales en un periodo de unos 3000 años (2000 a.C. al 1000 d.C). La cultura Maya realizo con éxito el manejo de los recursos naturales, en particular la flora, lo que conlleva a una gran diversidad de especies vegetales y los diversos sistemas de producción agroforestal, entre éstos los cultivos y el huerto familiar (Kantún-Balam et al., 2013). Cabe mencionar que la conservación del sitio arqueológico ha promovido la sucesión ecológica del bosque, así como el establecimiento de algunas especies arbóreas.

\section{CONCLUSIONES}

- Esta área protegida, Parque Arqueológico de Copán alberga flora nativa con un potencial en el uso medicinal, industrial y de aprovechamiento forestal. Sin embargo el sitio arqueológico se encuentran en un fragmento de bosque debido a las 
presiones antrópicas. También en el parque se registran especies con prioridad para su conservación, ya que algunas son enlistadas en la lista Roja de UICN y el apéndice II de CITES debido a la reducción de sus poblaciones así como por su comercialización.

- El estudio florístico del Parque Arqueológico de Copán es la base para evaluar la diversidad vegetal del área protegida como parte de una de las líneas estratégicas y del Programa de Manejo de Recursos Naturales propuesto en el plan de manejo 2014-2020.

\section{AGRADECIMIENTOS}

Se le agradece al Instituto en Arqueoastronomía y Patrimonio Cultural y Natural (IARPACUNA) de la Facultad de Ciencias Espaciales de la UNAH y la Asociación Copán por el apoyo logístico y económico. A los especialistas que colaboraron en la identificación de material y revisores anónimos.

\section{REFERENCIAS BIBLIOGRÁFICAS}

Asociación Copán (2013). Sitio Maya de Copán, plan de manejo 2014 - 2020. Copán Ruinas. Honduras $56 \mathrm{p}$.

Chi Quej, Jesús de los Ángeles. (2009). Caracterización y manejo de los huertos caseros familiares en tres grupos étnicos (Mayas peninsulares, Choles y Mestizos) del Estado de Campeche, México. Tesis de posgrado, Centro Agronómico Tropical de Investigación y Enseñanza, Turrialba, Costa Rica. 114 p.

Cordero, Jesús y Boshier David. (eds). (2003). Árboles de Centroamérica: un manual para extensionistas. Oxford Forestry Institute, Great Britain. Forestry Research Programme.

Flores José Salvador y Bautista Francisco. (2012). Knowledge of the Yucatec Maya in seasonal tropical forest management: the forage plants. Revista Mexicana de Biodiversidad 83: 503-518.

Gentry, Alwyn Howard. (1982). Patterns of Neotropical plant diversity. Evolutionary Biology 15: $1-84$.

Gómez-Domínguez, Héctor, Pérez Farrera, Miguel Ángel, Espinoza Jiménez, Josefa Anahí, y Marquez Reynoso, Mirna Ivett. (2015). Listado florístico del Parque Nacional Palenque, Chiapas, México. Botanical Sciences, 93(3), 559-578.

House, Paul Raymond, Lagos-Witte, Sonia, Ochoa, Lorena, Mejía, Thelma y Rivas Margarita. (1995). Plantas Medicinales Comunes de Honduras. 1ra. Edición. Tegucigalpa: 
Universidad Nacional Autónoma de Honduras. 555 pp.

House, Paul. (2007). Etnobotánica Maya, Parque Arqueológico Ruinas de Copán. Instituto Regional de Biodiversidad, IRBio, Centro Zamorano de Biodiversidad CZB, Asociación Copan, Instituto Nacional de Biodiversidad INBio, Instituto Hondureño de Antropología e Historia IHAH.26 p.

Kantún-Balam, Jesús, José Salvador-Flores, Juan Tun-Garrido, Jorge Navarro-Alberto, Luis Arias-Reyes y Jaime Martínez-Castillo. (2013). Diversidad y origen geográfico del recurso vegetal en los huertos familiares de Quintana Roo, México. Polibotánica, (36), 163-196.

Nelson Sutherland, Ciryl Hardy. (2008). Catálogo de las plantas vasculares de Honduras: espermatofitas. Tegucigalpa: Guaymuras y Secretaría de Recursos Naturales y Ambiente.

Rivas, Álvaro, Pablo Enrique Avendaño y Heimar Quintero. (2010). Updating peasant competencies to mitigate poverty in the Chorti community, Copán (Honduras). Agronomía Colombiana 28(3): 567-575.

Roque, José, Asunción Cano y Anita Cook. (2003). Restos vegetales del sitio arqueológico Casa Vieja, Callango (Ica). Rev. Peru. Biol. 10(1): 33-43.

UNESCO (2017). Maya Site of Copán. UNESCO World Heritage Centre. Recuperado de: http://es.unesco.org

Vega-López, Adrián, Juan Ignacio Valdez-Hernández, Víctor Manuel Cetina-Alcalá. (2003). Zonas ecológicas de Brosimum alicastrum Sw. en la costa del Pacífico mexicana. Madera y Bosque, 9(1):27-53.

Zamora-Crescencio, Pedro, José Salvador Flores Guido y Rocío Ruenes Morales (2009). Flora útil y su manejo en el cono sur del estado de Yucatán, México. Polibotánica 228: 227-250.

Zamora-Crescencio, Pedro, Celso Gutiérrez-Báez, William J. Folan, Ma. del Rosario Domínguez-Carrasco, Pascale Villegas, Geucilio Cabrera-Mis, Claudeth Marielli Castro-Angulo y Juan Carlos Carballo. (2012). La vegetación leñosa del sitio arqueológico de Oxpemul, municipio de Calakmul, Campeche, México. Polibotánica 33: 31-150.

Vargas Pérez, Pedro. (2004). El misterio maya. Revista InterSedes 5:1-16.

Vozzo, John A. (ed.). (2002). Tropical Tree Seed Manual: Part II, Species Descriptions. U.S. Department of Agriculture, Forest Service, Washington, D.C. 899 p. 


\section{ANEXO}

Cuadro 1. Lista de plantas vasculares registradas en el Parque Arqueológico de Copán.

Los nombres comunes son consultados en Nelson (2008), House et al. (1995) y las etiquetas de los especímenes depositados en los herbarios Cyril Hardy Nelson Sutherland (TEFH) y Paul C. Standley (EAP). Hábito: H. hierba; A. árbol; Ar. arbusto. Origen: N. nativa; I. introducida.

\begin{tabular}{|l|l|l|l|}
\hline Taxa & Habito & Origen & Nombre Común \\
\hline MONILOPHYTA & & & \\
\hline ADIANTACEAE & & & \\
\hline Adiantum trapeziforme L. & $\mathrm{H}$ & $\mathrm{N}$ & Canastilla \\
\hline MONOCOTYLEDONEAE & & & \\
\hline ARACEAE & & & \\
\hline Syngonium angustatum Schott & $\mathrm{H}$ & $\mathrm{N}$ & Mano de León \\
\hline Syngonium hoffmannii Schott & $\mathrm{H}$ & $\mathrm{N}$ & \\
\hline ARECACEAE & & & \\
\hline Acrocomia mexicana Karw. ex Mart. & $\mathrm{H}$ & $\mathrm{N}$ & Coyol \\
\hline BROMELIACEAE & & & \\
\hline Bromelia karatas L. & $\mathrm{H}$ & $\mathrm{N}$ & Motate \\
\hline Bromelia pinguin L. & $\mathrm{H}$ & $\mathrm{N}$ & Piñuela \\
\hline Tillandsia schiedeana Steud. & $\mathrm{H}$ & $\mathrm{N}$ & \\
\hline CYCLANTHACEAE & & & \\
\hline Carludovica palmata Ruiz \& Pav. & $\mathrm{H}$ & $\mathrm{N}$ & Junco \\
\hline CYPERACEAE & & & \\
\hline Cyperus odoratus L. & & & \\
\hline
\end{tabular}




\begin{tabular}{|l|l|l|l|}
\hline Taxa & Habito & Origen & Nombre Común \\
\hline DIOSCOREACEAE & & & \\
\hline Dioscorea sp. & H & N & Ñame \\
\hline MARANTACEAE & & & \\
\hline Maranta arundinacea L. & H & I & Calisguate, yuquilla \\
\hline ORCHIDACEAE & & & \\
\hline Oncidium cebolleta (Jacq.) Sw. & H & N & hoja de cebolla \\
\hline Encyclia nematocaulon (A. Rich.) Acuña & H & N & Cebollina \\
\hline Oeceoclades maculata (Lindl.) Lindl. & H & In & Orquídea monja africana \\
\hline Cohniella ascendens (Lindl.) Christenson & H & N & \\
\hline Epidendrum cardiophorum Schltr. & H & N & \\
\hline Catasetum integerrimum Hook. & H & N & Pipa de vieja \\
\hline POACEAE & & & \\
\hline Lasiacis ruscifolia (Kunth) Hitchc. & H & N & Carrizo \\
\hline SMILACACEAE & & & \\
\hline Smilax spinosa Mill. & A & N & Zarzaparrilla \\
\hline ANGIOSPERMAS BASALES & & & \\
\hline ANNONACEAE & N & N & Guanábana \\
\hline Annona muricata L. & A & N & Suncuya \\
\hline Annona purpurea Moc. \& Sessé ex Dunal & A & I & \\
\hline Rollinia mucosa (Jacq.) Baill. & & & \\
\hline Sapranthus violaceus (Dunal) Saff. & & & \\
\hline ARISTOLOCHIACEAE & & & \\
\hline Aristolochia maxima Jacq. & & & \\
\hline
\end{tabular}




\begin{tabular}{|c|c|c|c|}
\hline Taxa & Habito & Origen & Nombre Común \\
\hline \multicolumn{4}{|l|}{ PIPERACEAE } \\
\hline Peperomia alata Ruiz \& Pav. & $\mathrm{H}$ & $\mathrm{N}$ & \\
\hline Piper aduncum L. & A & $\mathrm{N}$ & Anisillo, cordoncillo \\
\hline Piper amalago L. & AR & $\mathrm{N}$ & \\
\hline Piper marginatum Jacq. & AR & $\mathrm{N}$ & Santa María \\
\hline Piper oblique-ovatum Trel. & AR & $\mathrm{N}$ & \\
\hline \multicolumn{4}{|l|}{ EUDICOTYLEDONEAE } \\
\hline \multicolumn{4}{|l|}{ ANACARDIACEAE } \\
\hline Astronium graveolens Jacq. & $A$ & $\mathrm{~N}$ & Ronrón \\
\hline Spondias mombin L. & $A$ & $\mathrm{~N}$ & Jobo \\
\hline \multicolumn{4}{|l|}{ APOCYNACEAE } \\
\hline Stemmadenia alfaroi (Donn. Sm.) Woodson & $A$ & $\mathrm{~N}$ & \\
\hline Asclepias curassavica Griseb. & $\mathrm{H}$ & $\mathrm{N}$ & Viborán \\
\hline Rauvolfia tetraphylla L. & AR & $\mathrm{N}$ & Alcotán, comida de culebra \\
\hline $\begin{array}{l}\text { Tabernaemontana eubracteata (Woodson) } \\
\text { A.O. Simões \& M.E. Endress }\end{array}$ & A & $\mathrm{N}$ & \\
\hline \multicolumn{4}{|l|}{ ARALIACEAE } \\
\hline Dendropanax arboreus (L.) Decne. \& Planch. & $A$ & $\mathrm{~N}$ & Mano de León \\
\hline \multicolumn{4}{|l|}{ ASTERACEAE } \\
\hline Lepidaploa tortuosa (L.) H. Rob. & AR & $\mathrm{N}$ & Flor de campo \\
\hline Eupatorium macrophyllum L. & $\mathrm{H}$ & $\mathrm{N}$ & Chilca, matapulgas \\
\hline \multicolumn{4}{|l|}{ BIGNONIACEAE } \\
\hline Mansoa hymenaea (DC.) A.H. Gentry & $\mathrm{L}$ & $\mathrm{N}$ & Bejuco de ajo \\
\hline
\end{tabular}




\begin{tabular}{|l|l|l|l|}
\hline Taxa & Habito & Origen & Nombre Común \\
\hline BORAGINACEAE & & & \\
\hline Cordia alliodora (Ruiz \& Pav.) Oken & A & $\mathrm{N}$ & Laurel \\
\hline Heliotropium indicum L. & $\mathrm{H}$ & $\mathrm{N}$ & Borraja, cola de alacrán \\
\hline BURSERACEAE & & & \\
\hline Bursera simaruba (L.) Sarg. & $\mathrm{A}$ & $\mathrm{N}$ & Jiote \\
\hline CACTACEAE & & & \\
\hline Epiphyllum hookeri Haw. & AR & $\mathrm{N}$ & Pitaya \\
\hline CAPPARACEAE & & & \\
\hline Crateva tapia L. & A & $\mathrm{N}$ & Manzana de playa \\
\hline Capparis frondosa Jacq. & AR & $\mathrm{N}$ & Hola ancha \\
\hline EUPHORBIACEAE & & & \\
\hline Jatropha curcas L. & AR & $\mathrm{N}$ & Piñón \\
\hline Croton heterochrous Müll. Arg. & AR & $\mathrm{N}$ & \\
\hline Ricinus communis L. & AR & $\mathrm{I}$ & Higuerilla \\
\hline FABACEAE & & & \\
\hline Acacia collinsii Saff. & A & $\mathrm{N}$ & Cachito \\
\hline Andira inermis (W. Wright) Kunth ex DC. & A & $\mathrm{N}$ & Almendro de rio \\
\hline Enterolobium cyclocarpum (Jacq.) Griseb. & $\mathrm{A}$ & $\mathrm{N}$ & Guanacaste \\
\hline Gliricidia sepium (Jacq.) Kunth ex Walp. & $\mathrm{A}$ & $\mathrm{N}$ & Madriado \\
\hline Pithecellobium dulce (Roxb.) Benth. & $\mathrm{A}$ & $\mathrm{N}$ & Mangollano \\
\hline Senna siamea (Lam.) H.S. Irwin \& Barneby & $\mathrm{A}$ & $\mathrm{N}$ & Acacia amarilla \\
\hline Indigofera suffruticosa Mill. & & & \\
\hline
\end{tabular}




\begin{tabular}{|c|c|c|c|}
\hline Taxa & Habito & Origen & Nombre Común \\
\hline Lonchocarpus rugosus Benth. & A & $\mathrm{N}$ & Coyote, chaperno \\
\hline Hymenaea courbaril L. & $\bar{A}$ & $\mathrm{~N}$ & Guapinol \\
\hline Diphysa americana (Mill.) M. Sousa & A & $\mathrm{N}$ & $\begin{array}{l}\text { Guachipilin, quebracho de } \\
\text { cerro }\end{array}$ \\
\hline \multicolumn{4}{|l|}{ FAGACEAE } \\
\hline Quercus sp. & A & $\mathrm{N}$ & Roble \\
\hline \multicolumn{4}{|l|}{ MALVACEAE } \\
\hline Ceiba pentandra (L.) Gaertn. & A & $\mathrm{N}$ & Ceiba \\
\hline Luehea speciosa Willd. & A & $\mathrm{N}$ & Guácimo negro \\
\hline Guazuma ulmifolia Lam. & A & $\mathrm{N}$ & Cablote \\
\hline \multicolumn{4}{|l|}{ MELASTOMATACEAE } \\
\hline Bellucia axinanthera Triana & AR & $\mathrm{N}$ & Manzanillo \\
\hline \multicolumn{4}{|l|}{ MELIACEAE } \\
\hline Cedrela odorata L. & A & $\mathrm{N}$ & Cedro \\
\hline Trichilia martiana C. DC. & A & $\mathrm{N}$ & Drago, Canelillo \\
\hline Swietenia macrophylla King & A & $\mathrm{N}$ & Caoba \\
\hline Trichilia havanensis Jacq. & A & $\mathrm{N}$ & Limoncillo \\
\hline \multicolumn{4}{|l|}{ MENISPERMACEAE } \\
\hline Cissampelos pareira L. & $L$ & $\mathrm{~N}$ & Alcotán \\
\hline \multicolumn{4}{|l|}{ MORACEAE } \\
\hline Brosimum alicastrum Sw. & A & $\mathrm{N}$ & Másica, San Ramón \\
\hline Castilla elastica Sessé & A & $\mathrm{N}$ & Hule \\
\hline
\end{tabular}




\begin{tabular}{|c|c|c|c|}
\hline Taxa & Habito & Origen & Nombre Común \\
\hline Maclura tinctoria (L.) D. Don ex Steud. & A & $\mathrm{N}$ & Mora colorada, mora \\
\hline Trophis racemosa (L.) Urb. & A & $\mathrm{N}$ & Campanilla \\
\hline Ficus velutina Humb. \& Bonpl. ex Willd. & A & $\mathrm{N}$ & \\
\hline \multicolumn{4}{|l|}{ MYRTACEAE } \\
\hline Eugenia sp. & AR & $\mathrm{N}$ & Guayabillo \\
\hline \multicolumn{4}{|l|}{ NYCTAGINACEAE } \\
\hline Pisonia aculeata L. & $\bar{L}$ & $\mathrm{~N}$ & Crucito, crucita \\
\hline \multicolumn{4}{|l|}{ PETIVERIACEAE } \\
\hline Petiveria alliacea L. & AR & $\mathrm{N}$ & Ipacina \\
\hline \multicolumn{4}{|l|}{ PRIMULACEAE } \\
\hline Ardisia compressa Kunth & $\mathrm{A}$ & $\mathrm{N}$ & Maca maca \\
\hline Ardisia paschalis Donn. Sm. & A & $\mathrm{N}$ & Madre monte \\
\hline $\begin{array}{l}\text { Bonellia macrocarpa (Cav.) B. Ståhl \& } \\
\text { Källersjö }\end{array}$ & AR & $\mathrm{N}$ & Naranjillo \\
\hline \multicolumn{4}{|l|}{ ROSACEAE } \\
\hline Rubus sp. & $\mathrm{AR}$ & $\mathrm{N}$ & mora \\
\hline \multicolumn{4}{|l|}{ RUBIACEAE } \\
\hline $\begin{array}{l}\text { Chrysophyllum mexicanum Brandegee ex } \\
\text { Standl. }\end{array}$ & $\mathrm{A}$ & $\mathrm{N}$ & Sálamo \\
\hline Genipa americana L. & A & $\mathrm{N}$ & Irayol \\
\hline Hamelia patens Jacq. & AR & $\mathrm{N}$ & Coralillo \\
\hline Psychotria pubescens Sw. & $\mathrm{AR}$ & $\mathrm{N}$ & Isaco de monte \\
\hline
\end{tabular}




\begin{tabular}{|c|c|c|c|}
\hline Taxa & Habito & Origen & Nombre Común \\
\hline \multicolumn{4}{|l|}{ SALICACEAE } \\
\hline Casearia corymbosa Kunth & A & $\mathrm{N}$ & Botoncillo \\
\hline Xylosma flexuosa (Kunth) Hemsl. & AR & $\mathrm{N}$ & Aguja de ara \\
\hline \multicolumn{4}{|l|}{ SAPINDACEAE } \\
\hline Thouinia serrata Radlk. & A & $\mathrm{N}$ & \\
\hline Allophylus punctatus (Poepp.) Radlk. & A & $\mathrm{N}$ & \\
\hline \multicolumn{4}{|l|}{ SAPOTACEAE } \\
\hline Sideroxylon capiri (A. DC.) Pittier & A & $\mathrm{N}$ & \\
\hline Chrysophyllum cainito L. & A & $\mathrm{N}$ & Caimito \\
\hline \multicolumn{4}{|l|}{ SIMAROUBACEAE } \\
\hline Alvaradoa amorphoides Liebm. & A & $\mathrm{N}$ & Zorra \\
\hline \multicolumn{4}{|l|}{ SOLANACEAE } \\
\hline Solanum erianthum D. Don & AR & $\mathrm{N}$ & Friega platos \\
\hline \multicolumn{4}{|l|}{ URTICACEAE } \\
\hline Cecropia peltata L. & A & $\mathrm{N}$ & Guarumo \\
\hline Urera baccifera (L.) Gaudich. ex Wedd. & AR & $\mathrm{N}$ & Chichicaste \\
\hline \multicolumn{4}{|l|}{ VERBENACEAE } \\
\hline Cornutia pyramidata L. & $\mathrm{A}$ & $\mathrm{N}$ & Manto de Jesús \\
\hline
\end{tabular}

